This item was submitted to Loughborough's Research Repository by the author.

Items in Figshare are protected by copyright, with all rights reserved, unless otherwise indicated.

\title{
Finite element simulations of ultrasonically assisted turning
}

PLEASE CITE THE PUBLISHED VERSION

http://dx.doi.org/10.1016/j.commatsci.2003.08.020

PUBLISHER

(c) Elsevier

VERSION

AM (Accepted Manuscript)

LICENCE

CC BY-NC-ND 4.0

REPOSITORY RECORD

Mitrofanov, A.V., V.I. Babitsky, and Vadim V. Silberschmidt. 2019. "Finite Element Simulations of Ultrasonically Assisted Turning". figshare. https://hdl.handle.net/2134/10847. 
This item was submitted to Loughborough's Institutional Repository (https://dspace.lboro.ac.uk/) by the author and is made available under the following Creative Commons Licence conditions.

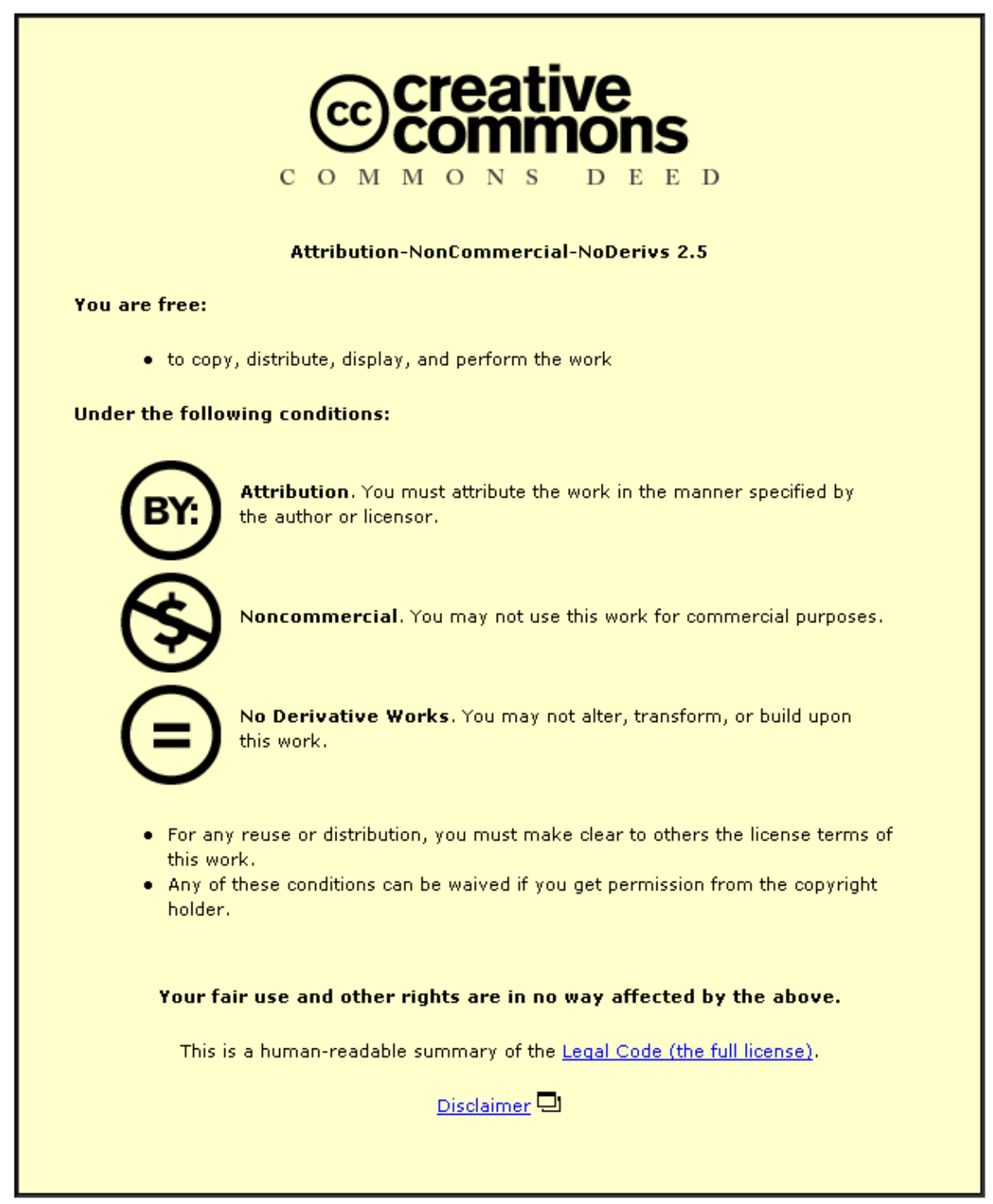

For the full text of this licence, please go to: http://creativecommons.org/licenses/by-nc-nd/2.5/ 


\title{
Finite element simulations of ultrasonically assisted machining
}

\author{
A. V. Mitrofanov", V. I. Babitsky, V. V. Silberschmidt \\ Wolfson School of Mechanical and Manufacturing Engineering, Loughborough University, \\ Loughborough, Leicestershire LE11 3TU, UK
}

\begin{abstract}
Ultrasonically assisted turning is a promising machining technology, where high frequency vibration ( $f \approx 20 \mathrm{kHz}$ ) with an amplitude $a \approx 10 \mu \mathrm{m}$ is superimposed on the movement of the cutting tool. Ultrasonic turning yields a noticeable decrease in cutting forces, heat and noise radiation, as well as a superior surface finish, comparing to the conventional machining technology. The present study utilizes both experimental techniques and numerical (finite element) simulations to analyze the microstructural processes at the cutting tool - chip interface. High-speed filming of the chip - tool interaction zone during cutting and microstructural and nanoindentation analyses of the machined surfaces are used to compare process zones and deformation processes for both conventional and ultrasonically assisted technologies. The suggested finite-element (FE) model, which utilizes MSC Marc/Mentat general FE code, provides a transient analysis for an elasto-plastic material, accounting for the contact interaction between a cutter and workpiece and material separation in front of the
\end{abstract}

\footnotetext{
* Corresponding author. Tel.: +44 1509 227566, fax: +44 1509 227648,

E-mail address: A.V.Mitrofanov@lboro.ac.uk
} 
cutting edge. A detailed analysis of cutting for a single cycle of ultrasonic vibration is carried out. Differences between conventional and ultrasonic turning in stress distribution in the process zone and contact conditions at the tool/chip interface are investigated.

PACS: 02.70.Dh; 81.05.Bx; 81.20.Wk.

Keywords: Ultrasonic vibration; Machining; Finite element method; Micromechanical analysis

\section{Introduction}

Machining is probably the most widespread material shaping process in mechanical manufacturing. This paper deals with one particular type of machining - turning. The technology involved in metal turning operations has advanced considerably in recent years. Modern methods of conventional turning, such as high speed machining, appear to be adequate for the cutting of engineering structural steels. Hence, the main activities in turning development are directed at improving machining characteristics for difficult to process materials. For example, nickel and titanium alloys, used in aerospace applications, cause high tool temperatures and subsequent fast wear of cutting edges even at relatively low cutting speeds. New methods of machining are also needed for ceramics, glass and other brittle materials, which presently require prolonged and expensive post-processing such as grinding and polishing to obtain the mirror-surface finish.

Ultrasonically assisted turning, with high frequency vibration ( $f \approx 20 \mathrm{kHz}$ ) with an amplitude $a \approx 10 \mu \mathrm{m}$ being superimposed on the movement of the cutting tool, allows significant improvements in machining of such materials. Ultrasonic turning demonstrates many advantages: a noticeable decrease in cutting forces and working temperatures for turning of hard materials $[1,2,3]$, improvement in surface finish by up to $50 \%$ compared to conventional technology [4], increase in tool life up to 20 times [1], noise reduction and 
successful implementation of ultrasonic turning for the machining of brittle plastics and glass $[5,6,7]$.

Dynamics and control of the ultrasonically excited cutting unit are investigated in $[2,9,10]$. However, little information is available on the mechanics of the ultrasonically assisted cutting process [2, 8, 9]. Initial studies of this process [8] contain some controversial data, which contradicts recent experimental results [4]. More recent papers, dealing with rheological models of elastic-rigid plastic material behavior, provide one with dynamic characteristics of ultrasonic vibration systems, such as the amplitude response of the cutting tool under technological load [2, 9]. Nevertheless, microstructural processes in the cutting tool workpiece interaction zone have not been investigated. Moreover, analytical modeling of material separation under the vibro-impact action of a cutter is hardly possible, due to the high level of the problem's complexity linked with its non-trivial three-dimensional geometry, caused by both a real shape of the cutter and, in a general case, non-orthogonal chip formation. Other complications of the problem are thermo-mechanical coupling and strain rate effects linked with the influence of the process temperature and deformation rates on material properties. The contact interaction and friction at the tool-chip interface, the influence of the material microstructure and impact loading conditions (caused by the cutter's vibrational movement) increase the complexity of the process. The inter-relation of all these factors determines mechanics of the ultrasonic cutting.

A holistic analysis of the process of ultrasonic turning necessitates a combination of experimental studies and numerical simulations of the cutter-workpiece interaction. Finite element analysis is the main computational tool in modeling cutting processes; it has been used to study turning for more than 30 years. A detailed overview of the development of FE models for turning operations can be found in [11, 12]. Earlier studies [13,14] dealt with a pre-formed chip, assuming its shape rather than predicting it, as well as neglecting initial 
stages of the process (i.e. chip separation and residual stresses) in simulations. The development of faster computers in 1980s allowed transient simulations of the entire cutting process from the first tool-chip contact to steady-state cutting conditions [15].

Updated Lagrangian formulation has been used in majority of publications since mid-1980s. In this approach elements move with the workpiece, thus experiencing large plastic deformation in the direct vicinity of the cutting tool's edge. For this approach, various criteria (material failure mechanisms) are introduced into modeling schemes in order to describe chip separation from the workpiece. These criteria are based on thresholds for the effective plastic strain [16], strain energy density [17], fracture strain (Johnson-Cook fracture model) [18, 19], and the critical distance from the cutting edge [20, 21]. Several algorithms have been used to implement the chip parting line: node separation along the predefined path $[15,16]$, element deletion [18, 19] and remeshing [22, 23, 24]. The majority of publications, based on commercial FE codes, deal with the continuous chip formation process. However, in some recent publications discontinuous or segmented chip formation is considered [19, 21, 25].

The diversity of mechanically treated materials as well as various magnitudes of technological parameters (cutting speeds, depths of cut, rake angles, etc.) resulting in the considerable variation of cutting forces and induced strains led to the introduction of different models of material behavior: elasto-plastic [17, 18] (including the multiplicative decomposition of elastic and plastic strains [26]), rigid-plastic [27] and elasto-visco-plastic [28].

The current stage of development of advanced FE schemes for turning is characterized by the introduction of additional process features into consideration, such as thermomechanical coupling and strain rate sensitivity, i.e. the influence of process temperature and deformation rates on material properties, and the frictional contact at the tool-chip interface, e.g. [19, 21, $22,28]$.

Still, to the authors' knowledge, no FE models for ultrasonic turning were suggested so far. 
The model of interaction between the workpiece and ultrasonically vibrating tool, using the experience accumulated in modeling of conventional turning, is introduced in this paper.

\section{Experimental results}

Existing experimental studies of ultrasonic cutting could be roughly divided into three main groups: (i) optimization of technological parameters of ultrasonic machining, such as directions, frequencies and amplitudes of vibration, (ii) analyses of cutting forces as compared to those of conventional machining, and (iii) surface profile measurements (see, for example [1, 4-7]). The scope of the present experimental investigation, in contrast, aims at the comparative analysis of the material microstructure after machining with ultrasonic and conventional technology. Microstructural analysis, nanoindentation tests and high-speed filming of the chip - tool interaction zone are used to study the processes of the tool-chip interaction and chip formation as well as the influence of cutting on the treated material.

A prototype of ultrasonically assisted cutting device with autoresonant control system has been designed at Loughborough University and utilized in experiments for turning Ni- and Tibase superalloys, widely used in the aerospace industry, as well as for many other materials. The experimental arrangement is described in detail in $[4,10]$.

Microscopic analyses of surface layers of the Ni-base superalloy Inconel 718, machined with ultrasonic vibration ( $f \approx 20 \mathrm{kHz}, a \approx 10 \mu \mathrm{m}$ ) in tangential direction and without it under the same cutting conditions (cutting speed $V=0.06 \mathrm{~m} / \mathrm{s}$, depth of cut $0.1 \mathrm{~mm}$ ), are carried out. Figure 1 shows a grain structure of the surface layers for Inconel 718 after conventional and ultrasonic turning. A transformed surface layer (about $25 \mu \mathrm{m}$ thick) is observed after conventional turning, whereas there are no visible alterations in the surface layer structure for ultrasonic turning. This qualitative comparison is supplemented with nanoindentation tests in order to obtain quantitative differences between microstructures of surface layers. According 
to the results of the nanoindentation analysis (performed with the NanoTest Platform made by Micro Materials Ltd, Wrexham, UK), the width of the transformed surface layer is two times smaller for the ultrasonically machined specimen than for the conventionally machined one (40 $\mu \mathrm{m}$ and $80 \mu \mathrm{m}$, respectively). Furthermore, the hardness of this layer for the ultrasonic technology is a half of that for conventional turning and considerably closer to the hardness of the untreated material (Fig. 2).

In another kind of experiments, a high-speed digital camera (Kodak Ektapro HS Motion Analyzer 4540) is used for real-time observations of chip - tool interaction zones during the cutting process (Fig. 3). The experiments have been conducted with various materials PMMA, mild steel and Inconel 718 - to study the influence of ultrasonic vibration on cutting of different types of materials, with the filming speed in the range from 9000 up to 27000 frames per second.

Significant differences between ultrasonic and conventional processes are observed for all tested materials. These differences manifest in specific features of the chip separation process, namely the size and shape of the process zone, and the type of the produced chip. The area of the visible process zone for ultrasonic turning and its width in the radial direction are considerably smaller than those for conventional turning. Deformation processes for the ultrasonic process are localized in the direct vicinity of the cutting edge along the surface of the workpiece and are not observed underneath the clearance face of the cutter at the newly formed surface layer, in contrast to the conventional turning process.

The chip produced by ultrasonically assisted turning is continuous with small serrations, whereas for the conventional process it is segmented. The curvature radius of chips is up to three times larger for the ultrasonic machining. In addition, various temper colors of chips produced by two technologies indicate the difference in temperatures during the ultrasonic and conventional cutting processes. 


\section{Finite element simulations}

Adequate computational analyses of the ultrasonic turning process should account for threedimensional geometry of the chip formation, material plasticity, thermomechanical coupling and strain rate effects, contact interaction and friction at the tool-chip interface, as well as the influence of the material microstructure. Incorporating all these features into the single FE model is a challenging task that should be implemented gradually increasing the complexity extent in order to validate the model at every stage of its development. A two-dimensional FE model considered at this stage of investigations utilizes MSC Marc/Mentat general FE code [29].

\subsection{Model description}

An orthogonal turning process with vibration in the direction of the cutting velocity is considered. Figure 4 shows a scheme of relative movements of the workpiece and cutting tool; the rotation axis of the cylindrical workpiece is orthogonal to the plane of the picture. Dimensions of the part of the workpiece used in FE simulations are $1.25 \mathrm{~mm}$ by $0.5 \mathrm{~mm}$ with the uncut chip thickness $t_{1}$ being $0.1 \mathrm{~mm}$. The model is developed under the plain strain assumption and initially consists of approximately 1000 four-node isoparametric elements with bilinear interpolation; the number of the elements increases during the simulation due to remeshing in the process zone to about 1700 . The characteristic size of the element in the process zone is $10 \mu \mathrm{m}$.

In the model the workpiece moves with a constant velocity, which corresponds to the cutting speed $V$ and equals $300 \mathrm{~mm} / \mathrm{s}$. Kinematic boundary conditions providing this type of movement are applied to the left, right and bottom sides of the workpiece, whereas its top surface is free:

$\left.V_{x}\right|_{\mathrm{AH}}=V,\left.V_{x}\right|_{\mathrm{FG}}=V,\left.V_{x}\right|_{\mathrm{HG}}=V,\left.V_{y}\right|_{\mathrm{AH}}=0,\left.V_{y}\right|_{\mathrm{FG}}=0,\left.V_{y}\right|_{\mathrm{HG}}=0$. 
The cutting tool (rake angle $\gamma=30^{\circ}$ ) is rigid and immovable (for simulations of the conventional turning process) or vibrates harmonically in the direction of cutting velocity in simulations of ultrasonic turning:

$u_{x}=-a \cos \omega t, \quad u_{y}=0$,

where $\omega=2 \pi f$, the frequency $f=20 \mathrm{kHz}$ and amplitude $a=13 \mu \mathrm{m}$.

The velocity of the cutting tip vibration is described by the relation $\dot{u}_{x}=v_{t} \sin \omega t$, where $v_{\mathrm{t}}=2 \pi a f \approx 1600 \mathrm{~mm} / \mathrm{s}$. Thus $v_{\mathrm{t}}>V$, providing a condition for separation of the cutter from the chip within each cycle of ultrasonic vibration.

The material modeled in all simulations is Inconel 718, which elasto-plastic behavior - in terms of the stress-strain relation - is described by the Ramberg-Osgood constitutive equation [30]:

$\sigma=\sigma_{\mathrm{Y}}+K_{\mathrm{Y}}\left(\varepsilon^{\mathrm{p}}\right)^{1 / M_{\mathrm{Y}}}$

where $\sigma_{\mathrm{Y}}$ is the elastic limit, $K_{\mathrm{Y}}$ is the coefficient of plastic resistance and $M_{\mathrm{Y}}$ is the hardening exponent. The stress-strain curve for Inconel 718 used in simulations is shown in Figure 5. In this paper thermal softening of Inconel 718 is neglected; the thermal dependence of its properties will be included in the coupled thermo-mechanical analysis at the next stage. The current model is considered to be strain-rate independent; however, the strain rate sensitivity will be introduced into the model as soon as reliable experimental data for Inconel 718 for strain rates of the order of $10^{5} \mathrm{~s}^{-1}$ will be available.

Direct experimental observations have shown that the level of friction is much less for ultrasonic turning due to the intermittent nature of the tool/chip contact under ultrasonic vibration, thus justifying the use of the frictionless contact interaction between the cutter and 
workpiece in the current model.

\subsection{Simulations and discussion of results}

Numerical simulations of conventional turning model the chip formation process from the initial contact between the cutter and workpiece at $t=0$ to a state of the fully formed chip $t=$ $0.00125 \mathrm{~s}$ (Fig. 6). The contour bands in the figure represent the equivalent von Mises stresses:

$\sigma_{\text {eq }}=\left(\frac{3}{2} s_{i j} s_{i j}\right)^{1 / 2}, s_{i j}=\sigma_{i j}-\frac{1}{3} \sigma_{k k} \delta_{i j}, \quad i, j, k=x, y, z$,

where $\sigma_{i j}$ are components of the Cauchy stress tensor, $\delta_{i j}$ is the Kronecker delta. The stress distribution changes during the initial stage of the simulation: the region occupied by stresses exceeding the yield stress propagates gradually along the surface of the workpiece and moves into the depth of the workpiece in the vicinity of the cutting tip. However, a steady-state stress distribution (as in Fig. 6d) is observed as soon as the chip is fully formed.

The time increment, chosen for the simulations of conventional turning is $2.5 \cdot 10^{-6} \mathrm{~s}$, thus the entire analysis till the transition to the steady-state behavior (in terms of stress levels in the process zone) requires 500 increments and takes approx. two hours of CPU time on HP 9000 workstation with 1 Gb RAM.

Due to the high-level deformations in the process zone in the direct vicinity of the cutting edge, the local level of equivalent plastic strain $\bar{\varepsilon}^{\mathrm{p}}=\int\left(\frac{2}{3} \dot{\varepsilon}_{i j}^{\mathrm{p}} \dot{\varepsilon}_{i j}^{\mathrm{p}}\right)^{1 / 2} d t, i, j=1,2,3$ exceeds unity, and elements in this zone could become highly distorted, leading to the accuracy degradation and even the analysis termination. To overcome this obstacle, an automatic remeshing procedure is used in the regions with a high level of elements' distortion. The 
remeshing of such zones of the workpiece and chip material is carried out either periodically (usually each 5 increments) or in the cases of considerable changes in the aspect ratios or angles of the finite elements.

Contrary to modeling of general cases of crack propagation in solids with a priori unknown paths, the separation of the material in the turning process occurs in the vicinity of the cutting edge, which moves along the kinematically prescribed straight line in two-dimensional simulations. Hence, the material separation in front of the cutting edge occurs along a predefined line and is currently subject to a critical stress criterion, i.e. material fails when nodal stresses reach a specified critical value.

Ultrasonic vibration is "switched on" at the stage of the fully formed chip, i.e. the simulation of conventional turning is restarted with the ultrasonically vibrating cutting tool. Figure 7 shows characteristic stages of a single cycle of vibration, which modeling takes 20 time increments $\left(2.5 \cdot 10^{-6} \mathrm{~s}\right.$ each $)$ for $f=20 \mathrm{kHz}$. The cutting process during the cycle of vibration could be divided into four main stages. During the first stage the cutter approaches the chip, in the second stage the cutter contacts the chip and starts penetrating into the workpiece causing the chip separation. The attainment of the maximum penetration depth is characterized by the highest level of generated stresses in the process zone and marks the end of the second stage. The following stage is unloading: the tool moves backwards and remains in contact with the chip even after the moment when the cutter's speed exceeds the cutting speed (due to the elastic spring-back). During this phase the relaxation of elastic strains in the process zone takes place. The last stage, starting with the full separation of the cutting edge from the chip, is the withdrawal of the cutter from the chip.

The FE analysis shows formation of the continuous chip, which is in good agreement with the results of high-speed filming experiments demonstrating a considerable reduction in chip segmentation for transition from the conventional cutting process to the ultrasonically assisted 
one.

In contrast to the steady-state stress distribution in simulations of conventional turning, a transient stress distribution is observed for ultrasonic cutting. During the approaching and withdrawal stages of the ultrasonic cycle the region with high stresses is concentrated in the vicinity of the separation point and between this point and the cutting tip. As soon as the cutter comes into the contact with the chip, the zone with equivalent stresses more than 800 MPa starts propagating from the cutting tip towards the chip's backside (along the BE direction, Fig. 4), occupying a band with the mean thickness of $60 \mu \mathrm{m}$. At the beginning of the unloading stage of the cycle of vibration the zone with high stresses begins shrinking in the direction of the cutting tip, gradually diminishing to the size it had at the approaching stage (i.e. before the tool contacts the chip). During the withdrawal stage the stress distribution in the process zone remains nearly unchanged. Hence, the greatest level of stresses throughout the cycle of vibration is attained during the penetration stage, whereas the stresses are significantly smaller in the rest of the cycle.

An important point is that the stress distribution in the process zone during the penetration phase of the cycle of ultrasonic vibration (Fig. 7b) is similar to the steady-state stress distribution in conventional turning (Fig. 6d). Hence, during the considerable part of the ultrasonic cycle stresses are noticeably lower than those in conventional turning. Consequently, mean magnitudes of stresses and, more generally, cutting forces are considerably less in ultrasonic turning. This correlates well with the reported experimental results [e.g. 1, 2].

Another important feature of ultrasonic cutting is the non-permanent character of the contact between the cutter and workpiece surface: the cutter remains in contact with the chip only during the penetration and unloading phases making it about $40 \%$ of the cycle of vibration (Fig. 8). Obviously, this amount depends on the ratio between the cutting speed and cutting 
tip velocity. The biggest attained length of the contact $L_{\mathrm{c}}$ (Fig. 4) between the chip and the cutter within the cycle of vibration is $80 \mu \mathrm{m}$ (Fig. 8), which is equal to the steady-state value of $L_{\mathrm{c}}$ in conventional cutting. Different contact conditions between the cutter and chip in ultrasonic and conventional cutting influence thermal boundary conditions and friction.

Residual strains in the machined layer are estimated after the end of the simulations of conventional turning. Figure 9 shows the dependence of equivalent plastic strain $\bar{\varepsilon}^{\text {p }}$ on the distance from the surface. Residual strains in the machined layer diminish more than fivefold within $100 \mu \mathrm{m}$ from the surface. This agrees reasonably with the results of nanoindentation tests, indicating an $80 \mu \mathrm{m}$ hardened layer for conventional turning.

The chip thickness ratio $r$, that is the ratio of the uncut chip thickness to the deformed chip thickness $r=t_{1} / t_{2}$ is approximately 0.8 for both conventional and ultrasonic turning simulations. Hence, the shear plane angle $\phi$ (Fig. 4) can be estimated as $\phi=\operatorname{arctg}\left(\frac{r \cos \alpha}{1-r \sin \alpha}\right) \approx 50^{\circ}$, providing fair agreement with the well-known Merchant equation [31] (which ignores material hardening effects): $\phi=\pi / 4-(\lambda-\alpha) / 2=60^{\circ}$ ( $\lambda$ is the friction angle and equals to zero for the frictionless contact).

Equivalent plastic strain rates $\left(\dot{\bar{\varepsilon}}^{\mathrm{p}}\right)$ observed in simulations of conventional turning attain the highest values in the shear plane (BE in Fig. 4) and occupy the band of the nearly constant width $30 \mu \mathrm{m}$ inclined at an angle $\phi^{*}=55^{\circ}$ to the $\mathrm{X}$-axis. The maximum magnitude of $\dot{\bar{\varepsilon}}^{\mathrm{p}}$ for conventional turning is about $10^{4} \mathrm{~s}^{-1}$. For simulations of ultrasonic turning $\dot{\bar{\varepsilon}}^{\text {p }}$ are the largest during the penetration stage and they are also concentrated in the shear plane. The maximum level of $\dot{\bar{\varepsilon}}^{\text {p }}$ for ultrasonic turning is observed in the vicinity of the cutting edge and attains approximately $10^{5} \mathrm{~s}^{-1}$. Hence, the maximum $\dot{\bar{\varepsilon}}^{\mathrm{p}}$ are about an order of magnitude higher in ultrasonic turning; this is explained by an increase in the maximum relative velocity between the chip and cutter due to the ultrasonic vibration of the latter. 


\section{Conclusions}

Experimental and numerical (finite element) investigations have been carried out to study the process of ultrasonically assisted turning in comparison with the conventional turning process. An elasto-plastic finite element model for ultrasonic turning was proposed as an enhancement of the model for conventional turning to explore microstructural processes at the cutting tool chip interface for both technologies. A detailed comparison based on numerical analyses of the transient stress distribution during the cycle of ultrasonic vibration and the steady-state stress distribution in conventional turning has shown that the mean level of stresses in the process zone and, consequently, cutting forces are considerably lower for the ultrasonically assisted technology, which correlates with known experimental results. In conventional turning the cutting tool stays in a permanent contact with the chip throughout the entire cutting process. In contrast, in ultrasonic turning the cutter remains in contact with the chip only about $40 \%$ of the time, according to FE simulations. Different contact conditions between the cutter and chip in ultrasonic and conventional cutting influence evolution of thermal processes and the friction type, causing differences in the chip formation observed experimentally in high-speed filming experiments. The next stage of investigations will be incorporation of additional features (thermo-mechanical coupling, frictional effects, etc.) into the finite element model of cutting processes.

\section{Acknowledgements}

Authors would like to thank Dr. G.K. Hargrave, Loughborough University, for developing and providing the optical part of the high-speed filming experiments.

\section{References}

[1] H. Weber, J. Herberger, R. Piltz, Annals of the CIPR, 33 (1984) 85. 
[2] V.K. Astashev, J. Machinery Manuf. Reliability, 5 (1992) 65.

[3] A. Markov, Ultrasonic machining of metals, Machinostroenie, Moscow, 1980 (in Russian).

[4] V.I. Babitsky, A.N. Kalashnikov, A. Meadows, A.A.H.P. Wijesundara, J. Mater. Process. Technol., 2003 (in print).

[5] J-D. Kim, I-H. Choi, Int. J. Adv. Manuf. Technol., 14 (1998) 2.

[6] D. Kremer, Mec. Ind. Mater., 48 (1995) 15.

[7] F. Klocke, O. Rubenach, Ind. Diamond Rev., 60 (2000) 227.

[8] J. Kumabe, Vibratory cutting, Dzikke Sjuppan, Tokyo, 1979 (in Japanese).

[9] V.K. Astashev, V.I. Babitsky, Ultrasonics, 36 (1998) 89.

[10] V.I. Babitsky, A.N. Kalashnikov, F.V. Molodtsov, Mechatronics (in print).

[11] T.H.C. Childs, K. Maekawa, T. Obikawa, Y. Yamane, Metal machining: theory and applications, Arnold, London, 2000.

[12] E.M. Trent, P.K. Wright, Metal Cutting, Butterworth-Heinemann, 2000.

[13] O.C. Zienkiewicz, The Finite Element Method in Engineering Science, :McGraw-Hill, London, 1971.

[14] T. Shirakashi, E. Usui, J. Japan Soc. Prec. Eng., 42 (1976) 340.

[15] J.S. Strenkowski, J.T. Carroll III, J. Eng. Ind., 107 (1985) 349.

[16] A. Behrens, B. Westhoff, Product Engng., 9 (2002) 13.

[17] Z.C. Lin, Y.Y. Lin, Theor. Appl. Fract. Mech., 35 (2001) 137.

[18] P.J. Maudlin, M.G. Stout, Minerals, Metals and Materials Society/AIME (USA), (1996) 29.

[19] E.-G. Ng, D.K. Aspinwall, Ind. Diamond Rev., 60 (2000) 305.

[20] T. Obikawa, E. Usui, Trans. ASME J. Manuf. Sci. Eng., 118 (1996) 208.

[21] M. Bäker, J. Rösler, C. Siemens, Comput. Struct., 80 (2002) 495. 
[22] T.D. Marusich, M. Ortiz, Int. J. Numer. Methods Eng., 38 (1995) 3675.

[23] D.R. Sandstrom, J.N. Hodowany, Machin. Sci. Technol., 2 (1998) 343.

[24] G.S. Sekhon, J.L. Chenot, Eng. Comput., 10 (1993) 31.

[25] T. Obikawa, H. Sasahara, T. Shirakashi, E. Usui, Trans. ASME J. Manuf. Sci. Eng., 119 (1997) 667.

[26] G.T. Camacho, T.D. Marusich, M. Ortiz, In: Proc. $4^{\text {th }}$ Int. Conf. on Computational Plasticity, Barcelona, Spain, 1995, p. 1835.

[27] E. Ceretti, P. Fallbohmer, W.T. Wu, T. Altan, J. Mater. Process. Technol., 59 (1996) 169.

[28] S. Lei, Y.C. Shin, F.P. Incropera, Int. J. Mach. Tools Manuf., 39 (1999) 731.

[29] MSC.Marc User’s Guide, Version 2001, MSC Software Corporation, Los Angeles, 2001.

[30] Military Handbook: Metallic Materials and Elements for Aerospace Vehicle Structures, Department of Defense, USA, 1997.

[31] M.E. Merchant, Mechanics of the metal cutting process, J. Appl. Phys., 16 (1945) 318. 


\section{Figure captions}

to the paper

"Finite element simulations of ultrasonically assisted machining"

by

\section{A. V. Mitrofanov, V. I. Babitsky, V. V. Silberschmidt}

Figure 1 Surface layers of Inconel 718 after conventional (a) and ultrasonic (b) turning

Figure 2 Comparative nanoindantation analyses of surface layers of Inconel 718 after ultrasonic and conventional turning. Indentation points are aligned along the radial direction starting from the surface of the specimen. The distance between two neighboring indents is $10 \mu \mathrm{m}$

Figure 3 Frames from high-speed filming of conventional (a) and ultrasonic (b) turning of the mild steel

Figure 4 A scheme of relative movements of the workpiece and cutting tool in orthogonal ultrasonically assisted turning

Figure 5 The stress-strain relation for Inconel 718

Figure 6 Conventional turning simulations: chip formation process and distribution of equivalent von Mises stresses: (a) $t=0$, (b) $t=0.00025 \mathrm{~s}$, (c) $t=0.00075 \mathrm{~s}$, (d) $t=0.00125 \mathrm{~s}$. The size of the frame is $0.7 \mathrm{~mm}$ by $0.6 \mathrm{~mm}$. A notch of 50 $\mu \mathrm{m}$ is introduced to improve the convergence at the beginning of the simulations.

Figure 7 Distribution of equivalent von Mises stresses for ultrasonic turning simulations at different moments of a single cycle of vibration: (a) cutter approaching the chip, (b) cutter in full contact with the chip, (c) and cutter moving away from the chip 
Figure 8 Simulated contact lengths $L_{\mathrm{c}}$ for conventional and ultrasonic cutting. Stages of the cycle of vibration for ultrasonic turning: approaching (I), penetration (II), unloading (III) and withdrawal (IV)

Figure 9 Residual plastic strains in the machined surface layer after conventional turning (FE results) 

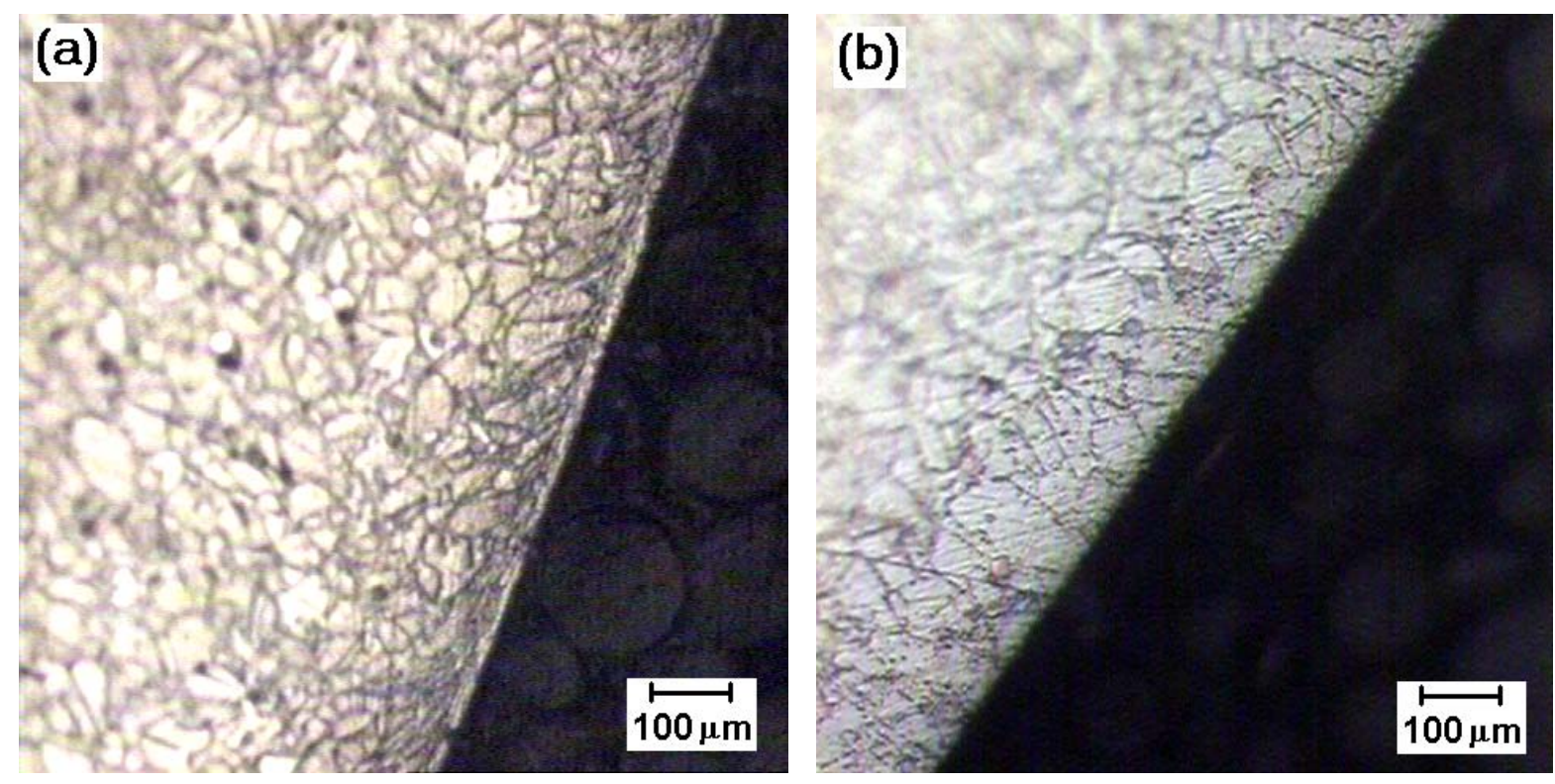

Figure 1

A. V. Mitrofanov, V. I. Babitsky, V. V. Silberschmidt

Finite element simulations of ultrasonically assisted machining 


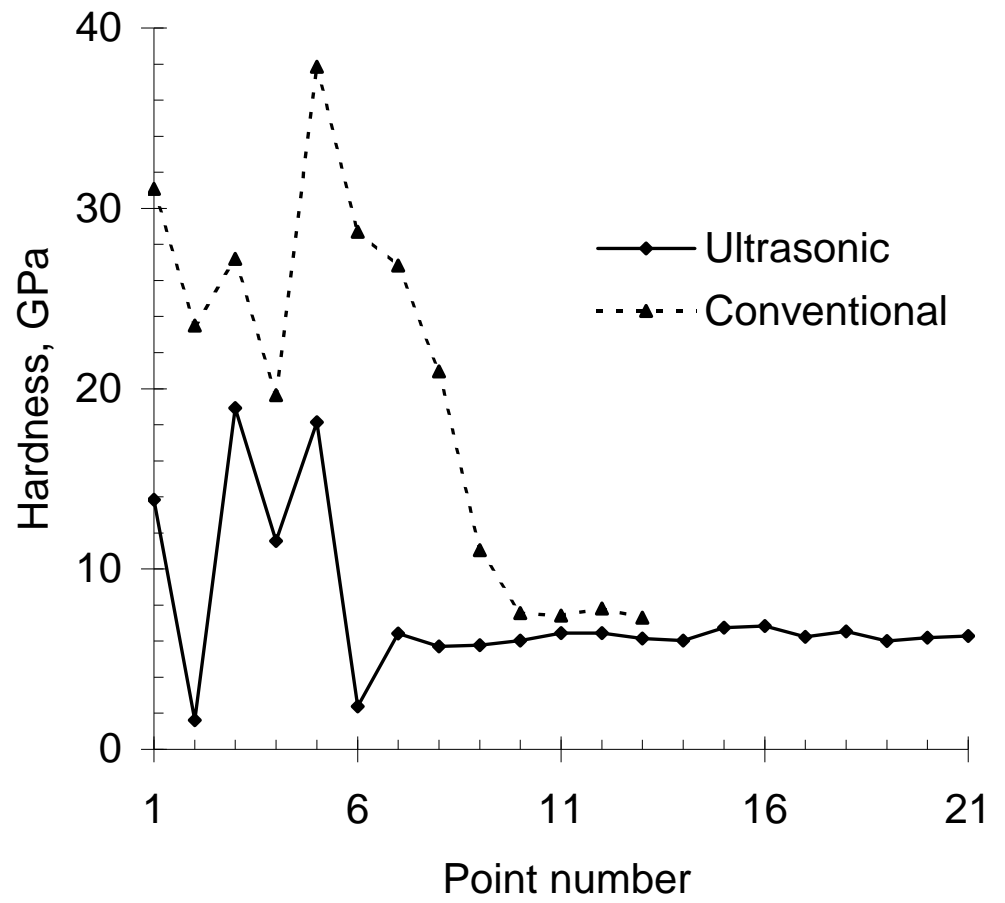

Figure2

A. V. Mitrofanov, V. I. Babitsky, V. V. Silberschmidt

Finite element simulations of ultrasonically assisted machining 

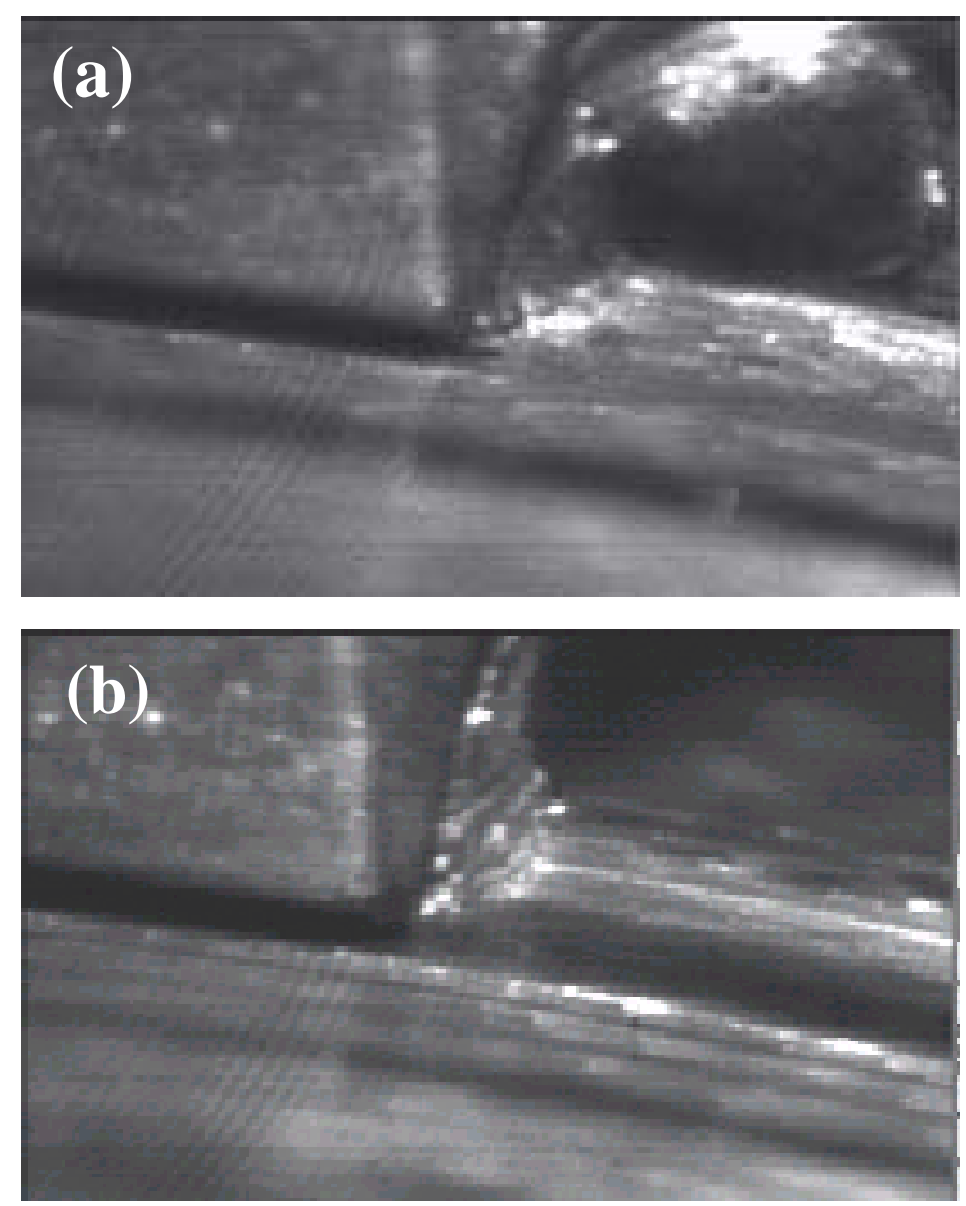

Figure 3

A. V. Mitrofanov, V. I. Babitsky, V. V. Silberschmidt

Finite element simulations of ultrasonically assisted machining 


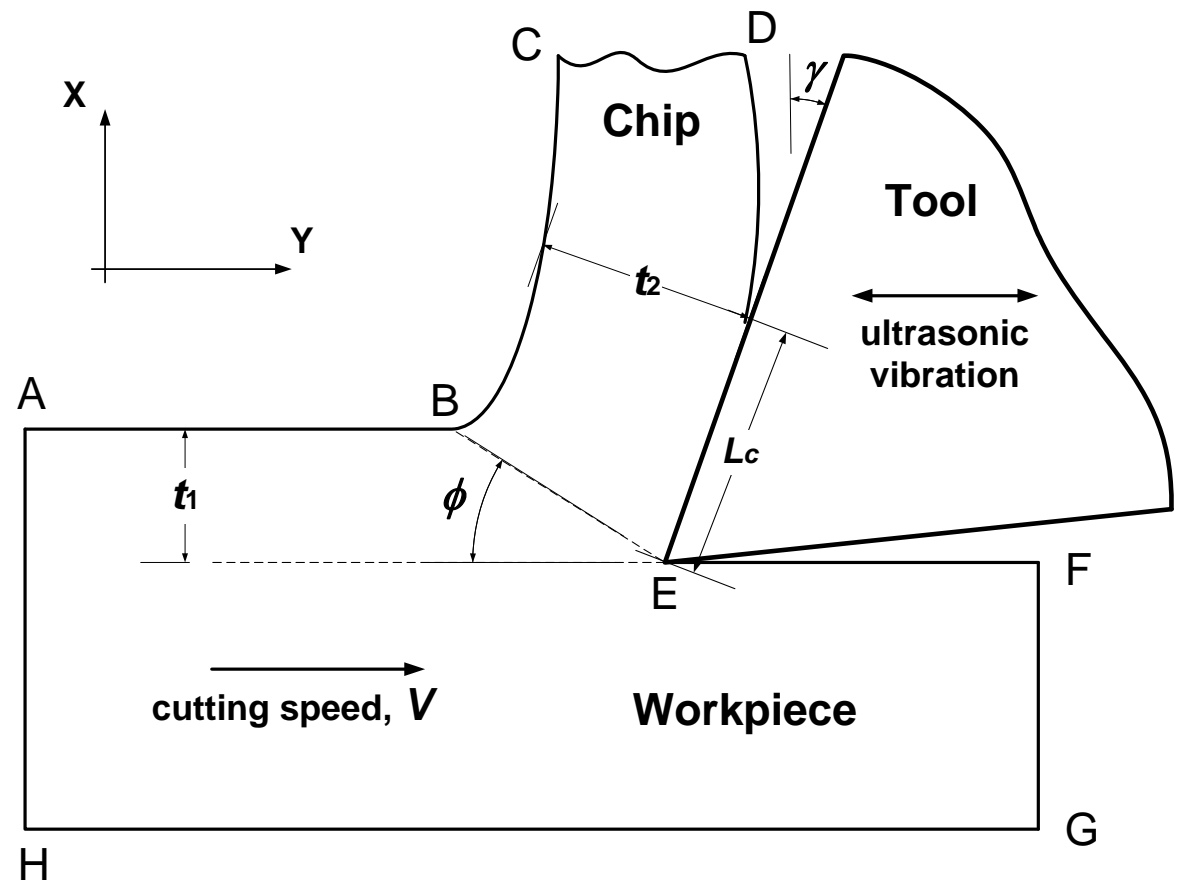

Figure 4

A. V. Mitrofanov, V. I. Babitsky, V. V. Silberschmidt

Finite element simulations of ultrasonically assisted machining 


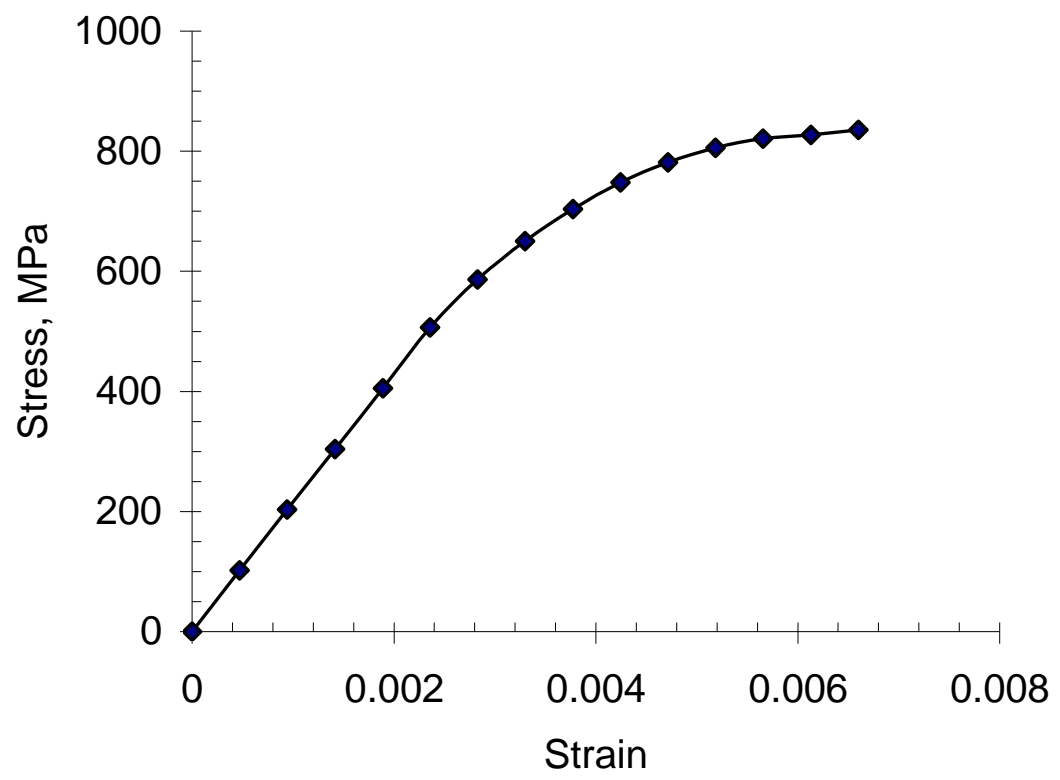

Figure 5

A. V. Mitrofanov, V. I. Babitsky, V. V. Silberschmidt

Finite element simulations of ultrasonically assisted machining 

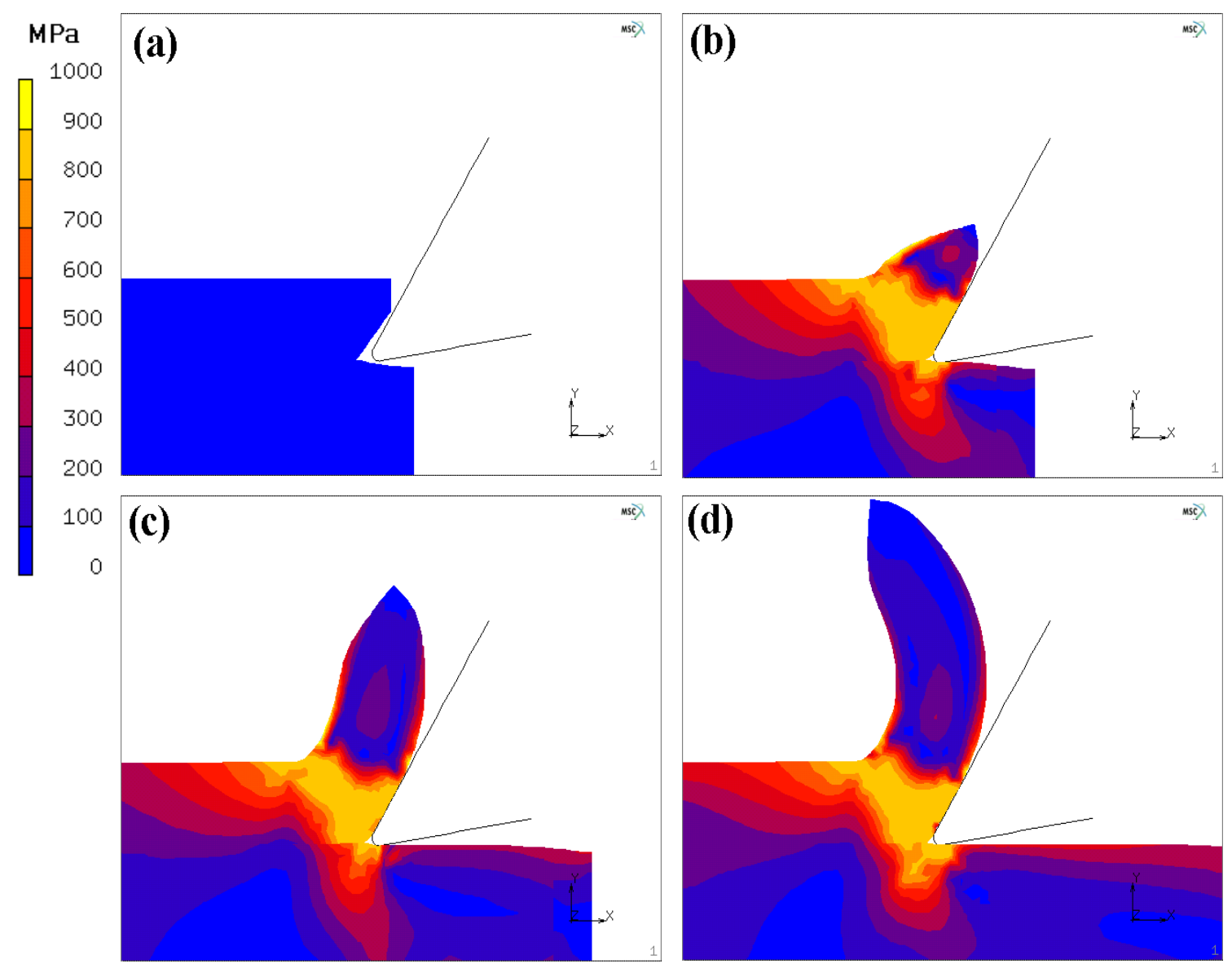

Figure 6

A. V. Mitrofanov, V. I. Babitsky, V. V. Silberschmidt

Finite element simulations of ultrasonically assisted machining 


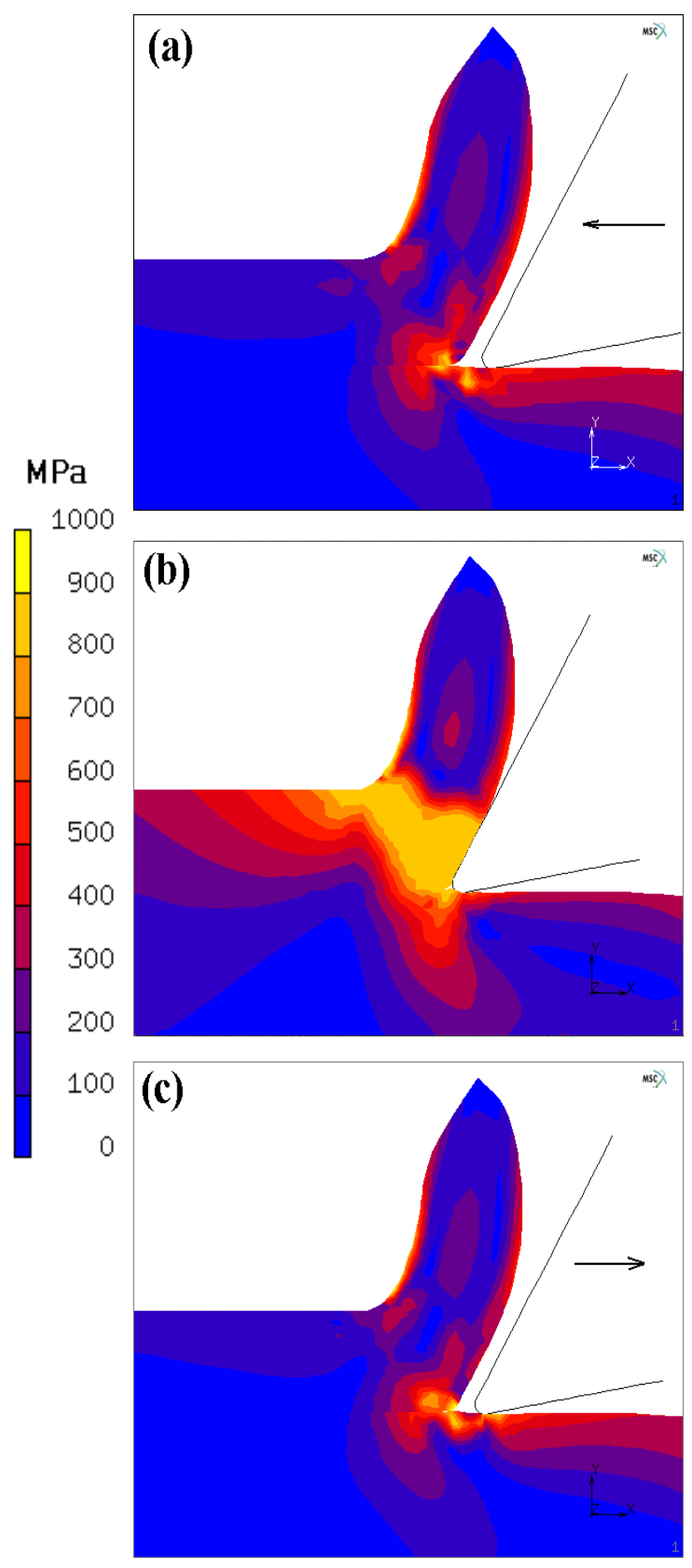

Figure 7

A. V. Mitrofanov, V. I. Babitsky, V. V. Silberschmidt

Finite element simulations of ultrasonically assisted machining 


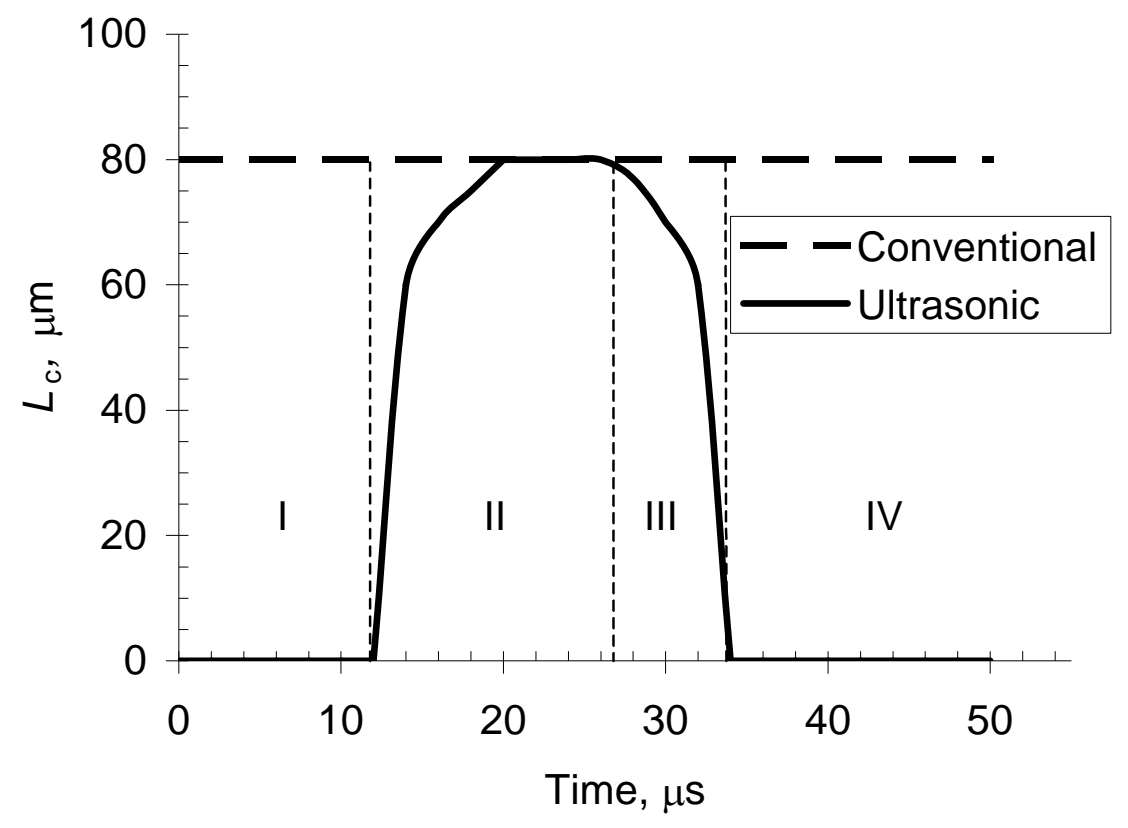

Figure 8

A. V. Mitrofanov, V. I. Babitsky, V. V. Silberschmidt

Finite element simulations of ultrasonically assisted machining 


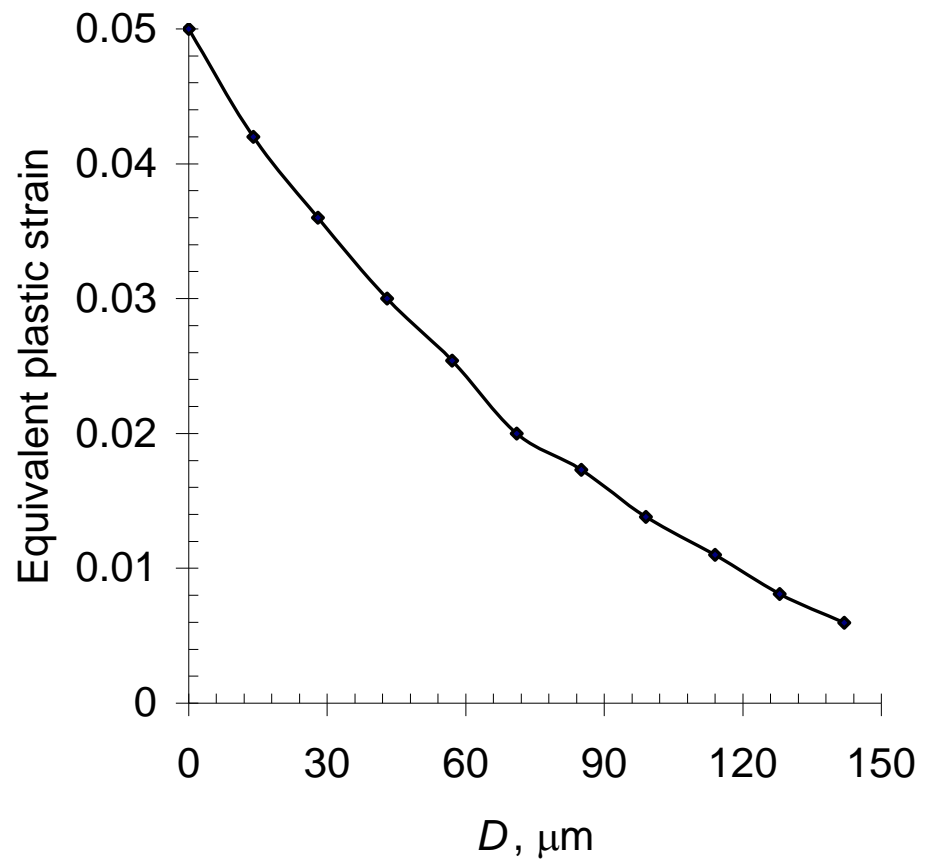

Figure 9

A. V. Mitrofanov, V. I. Babitsky, V. V. Silberschmidt

Finite element simulations of ultrasonically assisted machining 